\title{
Could targeting neighboring bacterial populations help combat bacterial vaginosis?
}

\author{
Nuno Cerca*,1 id \\ ${ }^{1}$ Centre of Biological Engineering (CEB), Laboratory of Research in Biofilms Rosário Oliveira, University of Minho, Campus de \\ Gualtar, Braga, Portugal \\ *Author for correspondence: Tel.: +351 253 60443; Fax: +351253 678 986; nunocerca@ceb.uminho.pt
}

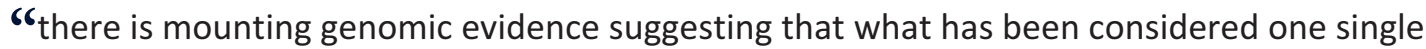 species can, in fact, be distinct bacterial species of the genus Gardnerella"

First draft submitted: 13 February 2019; Accepted for publication: 15 February 2019; Published online: 11 March 2019

Keywords: antimicrobial tolerance $\bullet$ bacterial vaginosis $\bullet$ biofilms $\bullet$ clue cells $\bullet$ multi-species communities

Bacterial vaginosis (BV), the most common vaginal disorder in women of reproductive age, remains one of the most intriguing and controversial challenges in modern day clinical microbiology. High prevalence and relapse rates make this infection of paramount global importance [1]. Yet, despite its impact on women's health, its etiology is still unknown [2].

In the early years, researchers and physicians attributed Gardnerella vaginalis as the sole causative agent of $\mathrm{BV}$ [3] but this concept was replaced by the polymicrobial theory, which proposed that BV was caused by a complex microbial community, where G. vaginalis was present, but not sufficient to cause BV [4]. A few years later, inconsistencies with the polymicrobial theory arose from epidemiological data, suggesting that BV was a sexually transmitted disease (STD), which would require a sole etiological agent [5]. The STD theory also had its pitfalls with some inconsistencies noted [6]. Furthermore, it has been suggested that BV is perhaps not a typical STD, but that sexual activity does enhance its occurrence [7], although no experimental data pursued this line of thought. Other theories have explored the inherent genetic background of women as a possible direction for the cause of BV [8]. However, once again, genotyping studies did not always agree with epidemiological data [9]. A recent hallmark in this research field was the realization that BV is associated with bacterial biofilms [10]. This puts all previous BV research into perspective.

\section{How biofilms might impact disease progression}

It is now widely recognized that planktonic cell growth does not accurately reflect bacterial growth in nature or in infectious diseases, where most bacteria grow as biofilms [11]. Biofilms are communities of bacteria attached to a surface and surrounded by a complex matrix that provides protection against environmental stresses [12]. In BV, this definition can fit the description of clue cells. Of relevance, biofilms have been described as a major cause of persistent infections, refractory to antimicrobial therapy [13]. This may be explained by the significant differences found between planktonic and biofilm growth modes [14]. Not surprisingly, the first study comparing tolerance profiles between planktonic cultures and biofilms formed by $G$. vaginalis concluded that biofilm cultures were more tolerant to hydrogen peroxide and lactic acid, two naturally occurring antimicrobial compounds existing in the vaginal environment [15]. Interestingly, this higher tolerance to antimicrobials shown by biofilm cells has already been demonstrated in vivo [16]. Thus, the current paradigm is that biofilm formation during BV can easily explain the high recurrence rates found worldwide [17].

\section{How current therapies are addressing the biofilm phenomenon}

Despite the advances of recent decades, current treatment is still focused on alleviating or eliminating symptoms through reduction of BV-associated bacteria [18]. This causes high levels of recurrence that can easily be explained by incomplete eradication of the biofilm. Nevertheless, there is now more academic interest in addressing the biofilm

Future $\because$ Medicine 
phenomenon regarding treatment options against BV biofilms [19,20]. Most of the in vitro studies are focused on $G$. vaginalis biofilms. This is not surprising since it has been shown before that the BV multi-species biofilms are mainly composed by G. vaginalis [10,21]. Furthermore, many of the less prevalent species found in the BV biofilm lack key virulent traits [22], therefore, making G. vaginalis a prime suspect as the etiological agent of BV. Traditionally, fair criticism against this hypothesis originates from the fact that some healthy women are colonized by G. vaginalis but never develop BV [23]. However, there is mounting genomic evidence suggesting that what has been considered one single species can, in fact, be distinct bacterial species of the genus Gardnerella [24]. In vitro evidence supports this since $G$. vaginalis isolated from healthy women lack the relevant traits needed to develop an early stage biofilm in the vaginal epithelium [25]. Future research will soon clarify if, in fact, there are virulent and avirulent Gardnerella spp.

Recently, a study utilized lysozyme to enhance the efficacy of common antibiotics, through induced degradation of a G. vaginalis biofilm [26], further highlighting the role of the biofilm in enhanced antimicrobial tolerance. As such, the search for anti-G. vaginalis biofilm agents is now thriving [27]. Efforts are also being directed toward identifying molecules that could prevent biofilm formation by $G$. vaginalis [28], which could be another alternative to enhance the efficiency of current therapies. While these results are somewhat promising, their exclusion of the possible role of other anaerobes on the biofilm ecosystem may hamper its applicability in real-life situations.

\section{Could targeting neighboring bacterial populations help combat bacterial vaginosis?}

If we think about Koch's postulates, we can argue that the mere host colonization by a bacterial species does not implicate that species in the disease etiology. Considering current knowledge of BV, it is not possible to determine if the presence of multispecies biofilms is the cause or simply a consequence of BV. To address this challenging question, my research group has started to study how microbial interactions within dual-species biofilms might contribute to BV development [29-31]. Interestingly, a recent analysis of several in vitro combinations of mixed species biofilms concluded that, although synergy is often found, there is always one species that dominates the niche [32].

Following the hypothesis that G. vaginalis is key as the early colonizer during BV [33], we are using an in vitro model that first allows a $G$. vaginalis biofilm to be formed and then introduces a second BV-associated species. On an earlier paper we have demonstrated that, in vitro, some tested species did enhance G. vaginalis mediated biofilm formation, but many others repressed it [30]. To follow, we further characterized the synergistic effects found in these dual-species biofilms: by combining G. vaginalis with 15 more BV-associated species, we observed, by confocal laser scanning microscopy, that those dual-species biofilms formed three unique morphotypes, with distinct spatial 3D organizations [31]. In biofilm morphotypes where G. vaginalis stays in the bottom layers of the biofilm and is covered by other species, a $G$. vaginalis oriented therapy will likely fail. This highlights the importance to include other species, somewhat abundantly found in the BV mixed species biofilms, in antimicrobial testing and development.

There is another reason why targeting other BV species should be of interest. In the same dual-species biofilms study mentioned above, we further analyzed how the 15 bacterial species influence gene expression in G. vaginalis. Although the panel of selected genes was very limited, we concluded that $v l y$, the gene encoding vaginolysin production, was significantly upregulated in the presence of Actinomyces neeui, Enterococcus faecalis and a few more bacterial species [31]. Vaginolysin is an endotoxin that can lyse vaginal cells' membranes contributing to the development of BV. This is of particular interest because previously, we demonstrated than when G. vaginalis shifts from planktonic to biofilm mode of growth, $v l y$ is repressed [14]. At first, this result could lead to a belief that biofilms would be preventing epithelial cells desquamation and the formation of clue cells. However, as in many other species, biofilm formation is a tightly time-dependent and regulated process. It is therefore conceivable that, during early colonization stages, $G$. vaginalis overcomes the resident flora and initiates a biofilm [25] without contributing to the most common symptoms of BV. However, when certain bacterial-bacterial interactions occur within the biofilm, a shift in G. vaginalis transcriptome occurs and BV symptoms develop. As we demonstrated before, not all bacterial species found in BV will play a pivotal role on this process [31]. By understanding which species play significant roles in BV development, novel antimicrobial strategies might be developed. If the early stage biofilm does not trigger specific molecular events, perhaps BV will not develop.

Hypothesis aside, the fact is that the field of BV biofilms is still in its infancy but has already highlighted key fundamental aspects of bacterial-bacterial interactions. Of course, what remains to be done is to improve in vitro studies to better mimic the in vivo situation and this is a big challenge that remains. 


\section{Financial \& competing interests disclosure}

The author has no relevant affiliations or financial involvement with any organization or entity with a financial interest in or financial conflict with the subject matter or materials discussed in the manuscript. This includes employment, consultancies, honoraria, stock ownership or options, expert testimony, grants or patents received or pending, or royalties.

No writing assistance was utilized in the production of this manuscript.

\section{References}

1. Kenyon C, Colebunders R, Crucitti T. The global epidemiology of bacterial vaginosis: a systematic review. Am. J. Obstet. Gynecol. 209(6), 505-523 (2013).

2. Reid G. Is bacterial vaginosis a disease? Appl. Microbiol. Biotechnol. 102(2), 553-558 (2018).

3. Gardner H, Dukes C. Haemophilus vaginalis vaginitis: a newly defined specific infection previously classified non-specific vaginitis. Am. J. Obstet. Gynecol. 69(5), 962-976 (1955).

4. Jung H-S, Ehlers MM, Lombaard H, Redelinghuys MJ, Kock MM. Etiology of bacterial vaginosis and polymicrobial biofilm formation. Crit. Rev. Microbiol. 43(6), 651-667 (2017).

5. Muzny CA, Schwebke JR. Pathogenesis of bacterial vaginosis: discussion of current hypotheses. J. Infect. Dis. 214(Suppl. 1), S1-S5 (2016).

6. Verstraelen H, Verhelst R, Vaneechoutte M, Temmerman M. The epidemiology of bacterial vaginosis in relation to sexual behaviour. BMC Infect. Dis. 10, 81 (2010).

7. Leppäluoto PA. Bacterial vaginosis: what is physiological in vaginal bacteriology? An update and opinion. Acta Obstet. Gynecol. Scand. 90(12), 1302-1306 (2011).

8. St John E, Mares D, Spear GT. Bacterial vaginosis and host immunity. Curr. HIV/AIDS Rep. 4(1), 22-28 (2007).

9. Turovskiy Y, Sutyak Noll K, Chikindas ML. The aetiology of bacterial vaginosis. J. Appl. Microbiol. 110(5), 1105-1128 (2011).

10. Swidsinski A, Mendling W, Loening-Baucke V et al. Adherent biofilms in bacterial vaginosis. Obstet. Gynecol. 106(5, Part 1), 1013-1023 (2005).

11. Donlan RM, Costerton JW. Biofilms: survival mechanisms of clinically relevant microorganisms. Clin. Microbiol. Rev. 15(2), 167-193 (2002).

12. Jefferson K. What drives bacteria to produce a biofilm? FEMS Microbiol. Lett. 236(2), 163-173 (2004).

13. Costerton JW, Stewart PS, Greenberg EP. Bacterial biofilms: a common cause of persistent infections. Science 284(5418), 1318-1322 (1999).

14. Castro J, França A, Bradwell KR, Serrano MG, Jefferson KK, Cerca N. Comparative transcriptomic analysis of Gardnerella vaginalis biofilms vs. planktonic cultures using RNA-seq. NPJ Biofilms Microbiomes 3(1), 3 (2017).

15. Patterson JL, Girerd PH, Karjane NW, Jefferson KK. Effect of biofilm phenotype on resistance of Gardnerella vaginalis to hydrogen peroxide and lactic acid. Am. J. Obstet. Gynecol. 197(2), 170.e1-170.e7 (2007).

16. Swidsinski A, Loening-Baucke V, Swidsinski S, Verstraelen H. Polymicrobial Gardnerella biofilm resists repeated intravaginal antiseptic treatment in a subset of women with bacterial vaginosis: a preliminary report. Arch. Gynecol. Obstet. 291(3), 605-609 (2015).

17. Muzny CA, Schwebke JR. Biofilms: an underappreciated mechanism of treatment failure and recurrence in vaginal infections: Table 1. Clin. Infect. Dis. 61(4), 601-606 (2015).

18. CDC. Sexually Transmitted Diseases Treatment Guidelines (2015). www.cdc.gov/std/tg2015/def ault.htm

19. Machado D, Castro J, Palmeira-de-Oliveira A, Martinez-de-Oliveira J, Cerca N. Bacterial vaginosis biofilms: challenges to current therapies and emerging solutions. Front. Microbiol. 6, 1528 (2016).

20. Bradshaw CS, Sobel JD. Current treatment of bacterial vaginosis—limitations and need for innovation. J. Infect. Dis. 214(Suppl. 1), S14-S20 (2016).

21. Hardy L, Jespers V, Dahchour $\mathrm{N}$ et al. Unravelling the bacterial vaginosis-associated biofilm: a multiplex Gardnerella vaginalis and Atopobium vaginae fluorescence in situ hybridization assay using peptide nucleic acid probes. PLoS ONE 10(8), e0136658 (2015).

22. Alves P, Castro J, Sousa C, Cereija TB, Cerca N. Gardnerella vaginalis outcompetes 29 other bacterial species isolated from patients with bacterial vaginosis, using in an in vitro biofilm formation model. J. Infect. Dis. 210(4), 593-596 (2014).

23. Cornejo OE, Hickey RJ, Suzuki H, Forney LJ. Focusing the diversity of Gardnerella vaginalis through the lens of ecotypes. Evol. Appl. 11(3), 312-324 (2018).

24. Cerca N, Vaneechoutte M, Guschin A, Swidsinski A. Polymicrobial infections and biofilms in women's health. Res. Microbiol. 168(9-10), 902-904 (2017).

25. Castro J, Alves P, Sousa C et al. Using an in vitro biofilm model to assess the virulence potential of bacterial vaginosis or non-bacterial vaginosis Gardnerella vaginalis isolates. Sci. Rep. 5(1), 11640 (2015). 
26. Thellin $\mathrm{O}$, Zorzi W, Zorzi $\mathrm{D}$ et al. Lysozyme as a cotreatment during antibiotics use against vaginal infections: an in vitro study on Gardnerella vaginalis biofilm models. Int. Microbiol. 19(2), 101-107 (2016).

27. Gottschick C, Szafranski SP, Kunze B et al. Screening of compounds against Gardnerella vaginalis biofilms. PLoS ONE 11(4), e0154086 (2016).

28. Algburi A, Zehm S, Netrebov V, Weeks R, Zubovskiy K, Chikindas ML. Benzoyl peroxide inhibits quorum sensing and biofilm formation by Gardnerella vaginalis 14018. Infect. Dis. Obstet. Gynecol. 2018, 1426109 (2018).

29. Castro J, Machado D, Cerca N. Escherichia coli and Enterococcus faecalis are able to incorporate and enhance a pre-formed Gardnerella vaginalis biofilm. Pathog. Dis. 74(3), ftw007 (2016).

30. Castro J, Cerca N BV and non-BV associated Gardnerella vaginalis establish similar synergistic interactions with other BV-associated microorganisms in dual-species biofilms. Anaerobe 36, 56-59 (2015).

31. Castro J, Machado D, Cerca N. Unveiling the role of Gardnerella vaginalis in polymicrobial bacterial vaginosis biofilms: the impact of other vaginal pathogens living as neighbors. ISME J. doi:10.1038/s41396-018-0337-0 (2019) (Epub ahead of print).

32. Raghupathi PK, Liu W, Sabbe K, Houf K, BurmØlle M, SØrensen SJ. Synergistic interactions within a multispecies biofilm enhance individual species protection against grazing by a Pelagic protozoan. Front. Microbiol. 8, 2649 (2018).

33. Machado A, Cerca N. Influence of biofilm formation by Gardnerella vaginalis and other anaerobes on bacterial vaginosis. J. Infect. Dis. 212(12), 1856-1861 (2015). 